\title{
Towards Automatic Phone-to-Phone Communication for Vehicular Networking Applications
}

\author{
Shaohan $\mathrm{Hu}^{*}$, Hengchang $\mathrm{Liu}^{\dagger}, \mathrm{Lu} \mathrm{Su}{ }^{\ddagger}$, Hongyan Wang*, Tarek F. Abdelzaher*, \\ Pan Hui ${ }^{\S}$, Wei Zheng $₫$, Zhiheng Xiell, and John A. Stankovic \\ ${ }^{*}$ University of Illinois at Urbana-Champaign, USA, ${ }^{\dagger}$ University of Science and Technology of China, \\ ${ }^{\ddagger}$ State University of New York at Buffalo, USA, ${ }^{\text {I University of Wisconsin-Madison, USA, }}$ University of Virginia, USA,

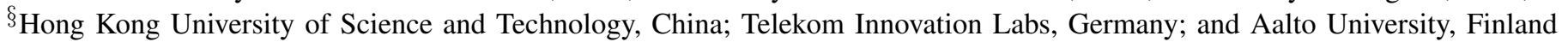 \\ Email: shu17@illinois.edu, hcliu@ustc.edu.cn, lusu@buffalo.edu, wang44@illinois.edu, zaher@cs.uiuc.edu, \\ panhui@cse.ust.hk, 18.weizheng@gmail.com,zx3n@virginia.edu, stankovic@cs.virginia.edu
}

\begin{abstract}
This paper explores direct phone-to-phone communication (via WiFi interface) among vehicles to support mobile sensing applications. Direct communication among drivers' phones is important in improving data collection efficiency and sharing participatory sensing information in an inexpensive manner. We design a practical and optimized communication mechanism for direct phone-to-phone data transfer among drivers' phones that strategically enables phone-to-phone and/or phone-toWiFiAP communications by optimally toggles the phone between the normal client and the hotspot modes. We take advantage of the WiFi hotspot functionality on smartphones, and hence require neither involvement of participants nor changes to existing wireless infrastructure and protocols. An analytical model is established to optimize toggling between client and hotspot modes for optimal system efficiency. We fully implement this system on off-the-shelf Google Galaxy Nexus and Nexus $S$ phones. Through a 35-vehicle 2-month deployment study, as well as simulation experiments using the real-world T-drive 9,211-taxicab dataset, we show that our solution significantly reduces data transfer delay time and maintains over $80 \%$ efficiency under varying system parameters. We even achieve $90 \%$ for parameter settings of the latest smartphones.
\end{abstract}

\section{INTRODUCTION}

This paper presents a practical mobile phone sensing system that utilizes direct phone-to-phone communication between vehicles to improve performance of mobile participatory sensing applications. Rather than designing a new protocol to improve vehicle-to-vehicle and vehicle-to-WiFiAP communications (e.g., see work on delay/disruption tolerant networks (DTN) [1]-[4], mobile ad-hoc networks (MANET) [5], [6], and vehicular networks [7]-[9]), we present an optimized phone-tophone communication scheme that uses only those capabilities exported to the user on today's smartphones. It strategically toggles between the normal (client) and hotspot modes on smartphones as would be needed to collect data from phones and upload to a remote back-end server. It does so without needing to root or jailbreak smartphones, which makes the functionality implementable as a third-party phone application. Moreover, it requires neither involvement of participants nor changes to existing wireless infrastructure and protocols.

This work is motivated by the proliferation of sensorequipped smartphones in the past few years. According to the International Data Corporation (IDC) Worldwide Quarterly Mobile Phone Tracker, it is estimated that 982 million smartphones will be shipped worldwide in 2015 [10]. The rich set of embedded sensors on smartphones makes mobile phone sensing an useful paradigm to support many applications that require real-time situation awareness, such as monitoring traffic congestion and commute delays. Vehicles are becoming popular as carriers of mobile sensing platforms for many reasons. First, their natural mobility increases coverage for many participatory and social sensing applications [11], [12]. Second, our daily commute itself has become a target of many research efforts, such as those that aim to save fuel consumption [13], find available parking positions [14], avoid traffic jams or routes in bad condition [15]-[17], or share general road-side events [18]. Research communities have recently investigated incentive mechanisms [19] to attract more smartphone users into mobile sensing, developed solutions to preserve participants' privacy [20], and addressed the sparse deployment problem [21] when mobile sensing systems do not have a sufficient number of participants.

Accordingly, we envision a new brand of sensing applications that use driver's phones to share mobile sensory data among vehicles as well as with infrastructure servers. We assume that users will exploit their cellular data bandwidth to download results from such servers, such as real-time traffic speed maps. However, they will typically not want the same mobile sensing applications to use their cellular communication for altruistic raw data upload to the server, since unlimited data plans are no longer prevalent [22], [23]. Instead, the paper explores a WiFi-based approach for uploading the sensor data needed for the service.

Wi-Fi based store and forward of sensed real-time data may result in a large latency [24], which motivates optimizing data transfer among vehicles as well as between vehicles and the infrastructure for faster offloading. Current communication techniques on smartphones that support peer-to-peer sharing, such as WiFi ad-hoc mode [25] and WiFi Direct [26], have significant limitations and are not directly usable for mobile sensing. WiFi ad-hoc is not supported on most popular phones unless rooted or jailbroken and will probably not be in the near future due to economic and political issues [27]. WiFi Direct was not designed with opportunistic networking in mind, but tries to connect WiFi enabled devices such as printers and cameras in a secure way and as easily as possible. User involvement is mandatory for WiFi Direct for security reasons [28]. Also note that even if WiFi Direct can overcome its mentioned limitations in the near future, the phones still need to switch between the WiFi Direct "peer mode" (to connect directly with other peers also in the peer mode) and the normal WiFi client mode (to connect to WiFi APs), as a phone in peer mode is not able to connect to normal WiFi APs to offload data. Thus our method actually generalizes to cover the WiFi Direct type of scenarios in the future.

In contrast, we utilize a WiFi hotspot switching approach 
that is compatible with existing WiFi APs as the functionalities needed are supported by the standard Android API and Java Reflection, which does not require users to root or jailbreak smartphones. Two phones can establish connections when one of them is in the hotspot mode and the other in the client mode, and a phone can offload data to access points when in the client mode. Initial efforts provided proof-of-concept prototypes [28]. Two important questions remain unanswered: first, is automatic phone-to-phone data transfer achievable in a highly mobile vehicular environment? Second, how to switch between the hotspot and client modes in an efficient way in order to minimize the expected wasted time due to phones being in incompatible modes? Our paper addresses the above questions, and makes the following contributions.

- To the best of our knowledge, this is the first fully deployed smartphone-based vehicular mobile sensing system in which automatic phone-to-phone communication is achieved and is compatible with existing wireless infrastructure. While social sensing regarding traffic and daily commutes provides the motivating applications, this paper is strictly about the mobile communication platform needed to support such applications.

- An analytical model is established to optimize system parameters in an adaptive fashion to achieve high system efficiency. We also provide empirical results to support several important design decisions in our system.

- We evaluate our analytical model and demonstrate the performance of our system by providing results from a real 35-participant 2-month deployment using Google Android phones, as well as simulation experiments using T-drive 9,211-taxicab dataset [29], [30]. Results show that our solution significantly reduces data transfer delay time and maintains above $80 \%$ efficiency under varying system parameters, even achieving $90 \%$ for parameter settings of the latest smartphones.

The remainder of this paper is organized as follows. After discussing related work in Section II, we give detailed problem descriptions in Section III. We then present our analytical model and system designs in Section IV and V. We evaluate our system and solution in Section VI. And finally Section VII concludes.

\section{RELATED WORK}

Prior work on vehicular mobile sensing and communication generally falls into one of two categories: either using phones for data collection and uploading (to back-end servers) without peer-to-peer communication; or using DTN- or MANET- style vehicle-to-vehicle communication but on dedicated hardware instead of phones. We are the first to offer a fully deployed system that leverages both phone-to-phone and phone-to-AP communications from vehicle-resident smartphones, customized for the needs of mobile sensing.

Several prior mobile social sensing applications leverage smartphones placed in vehicles. For example, the Nericell project [16] presents a system that performs rich sensing using smart phones that users carry with them in normal courses, to monitor road and traffic conditions. The GreenGPS system [13] provides a service that computes fuel-efficient routes for vehicles between arbitrary end-points, by exploiting vehicular sensor measurements available through the On Board Diagnostic (OBD-II) interface of the car and GPS sensors on smartphones. SignalGuru [15] is a software service that relies solely on a collection of mobile phones to detect and predict the traffic signal schedule, producing a Green Light Optimal Speed Advisory (GLOSA). These systems rely on WiFi access points, since transmitting data through cellular data networks is expensive. However, open public WiFi is becoming less prevalent as more access points are becoming private or secure. Our paper aims to overcome this drawback by allowing smart phones to exchange data in an opportunistic way to maximize upload opportunities.

Our application scenario requires moving wireless nodes and sometimes information processing in intermittentlyconnected networks. MANETs and DTNs are therefore important overlapping fields of research to our paper. For instance, CafNet [6] in the CarTel project [18] is a delaytolerant stack that enables mobile data muling and allows data to be sent across an intermittently connected network. The CafNet protocols allow cars to serve as data mules, delivering data between nodes that are otherwise not connected to one another. Similarly, the DieselNet testbed [5] consists of 35 buses, each with a Diesel Brick, which is based on a HaCom Open Brick computer. MultiNets [31] investigates the switching between WiFi and cellular modes on phones for energy and/or throughput considerations. It is, however, not suitable for our targeted vehicular mobile sensing/networking scenarios because of limited $\mathrm{WiFi}$ accessibility in outdoor environments and that we do not allow cellular data transmission due to the constant generation of potentially huge amount of sensory data. Other related work in this field include [1]-[4]. The main differences of our proposed system over this work are two-fold. First, most of them use data mules for data collections, instead, we systematically investigate the performance of realistic opportunistic networking via direct phone-to-phone communication, which is now possible with most popular mobile devices. Second, while they mainly focus on the optimization of communication stack to take advantage of short vehicle meeting times, we aim to leverage commonly open APIs on smartphones and hence restrict ourselves to what can be done with the available stacks.

Our work is also related to efforts in the vehicle networking community, called VANET, where the goal is usually to increase road safety and transport efficiency, and provide Internet access on the move to ensure wireless ubiquitous connectivity. Research challenges in evolving connected vehicle architecture, such as leveraging street parking to enable vehicular Internet access [7] and investigating application-driven interand intra-cluster communication in VANETs [8], has been deeply investigated. However, in mobile participatory sensing, the vehicle-to-vehicle communication problem targets a different goal: we aim to help participants who rarely approach wireless access points themselves to deliver their sensory data to the back-end server more quickly. There appears to be no straightforward solution in the VANET regime to provide automatic and efficient vehicle-to-vehicle communication with smartphones.

Finally, existing communication techniques on smartphones that support peer-to-peer sharing, such as WiFi ad-hoc [25] and WiFi Direct [26], have significant limitations and are not directly usable for social sensing. WiFi Ad-Hoc is still not supported on most popular phones unless rooted or jailbroken and will probably not be in the near future [27]. WiFi Direct is 


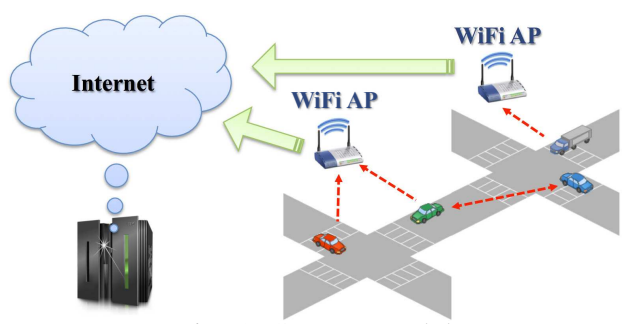

Fig. 1: System model

not designed with opportunistic networking in mind, but tries to connect WiFi enabled devices such as printers and cameras in a secure way and as easily as possible [28]. In addition, once a phone is set to $\mathrm{WiFi}$ ad-hoc or Direct mode to support peerto-peer communication, it is no longer able to connect to $\mathrm{WiFi}$ APs and offload data to the back-end servers. Our WiFi hotspot switching approach overcomes these drawbacks and does not need to root or jailbreak smartphones [28], however, there is still a lack of real deployment for performance evaluation especially in highly mobile environment, which incidentally is one main contribution of our paper as well.

\section{System Model \& Problem Description}

Our system is aimed to operate in a vehicular mobile sensing network where sensory data is generated and collected from participants' vehicle-resident smartphones, as illustrated in Figure 1. WiFi coverage is only sparsely available within the sensing area. When a car moves into the coverage area of a WiFi access point, the phone transmits its locally stored data to the back-end server via WiFi communications. In addition, we particularly allow phones to communicate with each other in order to reduce data transfer delays.

While a significant car density may be observed in an urban area, it may not be appropriate to assume that all or even a large portion of drivers are running our system on their phones. Instead, we make the more conservative assumption that only a small fraction of phones are running our system at any given time. Hence, it would be unusual for more than two such phones to be within each other's communication range at a time. Therefore, in this work we focus our analysis on pairwise encounters between phones, as opposed to optimizing general multi-party communications within phone clusters. To demonstrate the validity of our assumption, we record the number of vehicles in all meeting events in the T-drive dataset containing 9,211 taxicabs. We set the transmission range to be $30 \mathrm{~m}$, according to our own transmission tests using Google smartphones in vehicles. We find that pairwise encounters make up about $80 \%$ of all meeting occurrences. Considering that the scale of this dataset is already quite large, the ratio of pairwise encounters would further increase with less participants in realistic settings.

In our system, as a phone joins the vehicle network, it enters the client mode, in which it searches for available communication opportunities, with either a phone in hotspot mode or a WiFi AP. Meanwhile, a timer is started to control how long the phone can stay in client mode searching. When the timer expires, the phone switches itself to become a WiFi hotspot. The phone then listens for incoming connection attempts from other phones that are in client mode. Similarly, another timer is used to switch the phone back to client mode upon expiry.

In either client or hotspot mode, whenever the phone sees a communication opportunity, the timer pauses as the phone enters transmission mode, and the data exchange starts with the other party (phone or back-end server via WiFi AP). When the communication is terminated due to either data transmission completion or cars moving out of range, the phone goes back to its previous mode, with the timer resumed.

As two phones approach each other, if they are both in hotspot (or client) mode, they cannot communicate until one of them toggles mode. Similarly, when a phone enters an WiFi AP coverage area, it cannot offload data if it's in hotspot mode. Therefore, the time durations phones stay in each mode is crucial. Under our described model, we are then interested in solving the System Efficiency Optimization problem, where System Efficiency is defined to be, of the entire time duration that phones are within communication range with each other (or WiFi APs), the proportion of time when data transfer can actually take place. The problem is challenging because the information when vehicles meet each other or move into $\mathrm{WiFi}$ coverage area is NOT a priori. In the next section, we establish an analytical model for the optimal mode-toggling policy and provide our solution to optimize important system parameters.

\section{Analytical Formulation \& Solution}

In this section, we present the analytical formulation of the optimal mode-toggling policy for maximizing the total expected transmission duration in our targeted vehicular phoneto-phone networks.

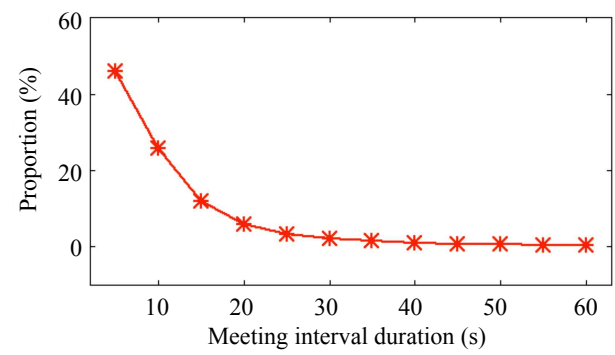

Fig. 2: Proportion of various meeting interval length from T-drive dataset.

We learn from preliminary experiments that connection rarely establishes in highway driving scenarios, regardless of whether the two cars are moving towards the same or opposite directions. On the other hand, when two cars meet and move toward the same direction in an urban or residential area, data transfer duration typically lasts quite long, which can also occur, for example, when the two cars close to each other park in the same parking lot or are caught in a traffic jam. Therefore, in these cases where the transmission duration is either extremely short or long, the switching of the phones' modes does not play a dominating role in system efficiency. Figure 2 shows the distribution of car meeting interval lengths within the T-drive dataset. We observe that around $46 \%$ of meeting events last less than 5 seconds, and less than $1 \%$ longer than 1 minute. Thus, more than $50 \%$ of car meeting events are around the middle of the distribution and potentially can benefit considerably from our system.

$t_{0}, \quad$ Hotspot: $r \quad t_{0}, \quad$ Client: $s$
Time Frame: $f=2 t_{0}+r+s$
Fig. 3: Hotspot-Client switching cycle

A complete cycle of the hotspot switching procedure is decomposed in Figure 3. As seen, a phone switches from 


\begin{tabular}{|c|c|c|c|c|}
\hline$t_{1}$ & $t_{2}$ & $t^{*}$ & $M_{1}$ & $E\left(M_{1}-t^{*}\right)$ \\
\hline$\left[0, t_{0}\right)$ & {$\left[0, t_{0}\right)$} & $\infty$ & $\sim$ & 0 \\
\hline \multirow[t]{3}{*}{$0, t_{0}$} & \multirow{3}{*}{$\begin{array}{l}{\left[t_{0}, t_{0}+r\right)} \\
\text { or } \\
{\left[0, t_{0}\right)}\end{array}$} & \multirow{3}{*}{$2 t_{0}+r-t_{2}$} & $M_{1} \geq t_{0}+r$ & $\frac{1}{2 f^{2}}\left[\left(M_{1}-t_{0}\right)^{2}-\frac{1}{3}\left(M_{1}-r\right)^{3}+\frac{1}{3}\left(M_{1}-t_{0}-r\right)^{3}\right]$ \\
\hline & & & $r \leq M_{1}<t_{0}+r$ & $\frac{1}{2 f^{2}}\left[\left(M_{1}-t_{0}\right)^{2} t_{0}-\frac{1}{3}\left(M_{1}-r\right)^{3}\right]$ \\
\hline & & & $t_{0} \leq M_{1}<r$ & $\frac{t_{0}}{2 f^{2}}\left(M_{1}-t_{0}\right)^{2}$ \\
\hline \multirow{2}{*}{$0, t_{0}$} & \multirow{2}{*}{$t_{0}+r, 2 t_{0}+r$} & \multirow{2}{*}{$\max \left(t_{0}-t_{1}, 2 t_{0}+r-t_{2}\right)$} & $M_{1} \geq t_{0}$ & $\frac{2}{f^{2}}\left(\frac{M_{1}}{2} t_{0}^{2}-\frac{1}{3} t_{0}^{3}\right)$ \\
\hline & & & $M_{1}<t_{0}$ & $\frac{1}{3 f^{2}} M_{1}^{3}$ \\
\hline \multirow{2}{*}{$0, t_{0}$} & \multirow{2}{*}{$2 t_{0}+r, f$} & \multirow{2}{*}{$t_{0}-t_{1}$} & $M_{1} \geq t_{0}$ & $\frac{1}{f^{2}}\left[\frac{1}{3} t_{0}^{3}-\frac{1}{2}\left(M_{1}+s\right) t_{0}^{2}+M_{1} s t_{0}\right]$ \\
\hline & & & $M_{1}<t_{0}$ & $\frac{1}{f^{2}}\left(\frac{1}{2} s M_{1}^{2}-\frac{1}{6} M_{1}^{3}\right)$ \\
\hline \multirow{2}{*}{$t_{0}, t_{0}+r$} & \multirow{2}{*}{$t_{0}, t_{0}+r$} & \multirow{2}{*}{$2 t_{0}+r-\max \left(t_{1}, t_{2}\right)$} & $M_{1} \geq r$ & $\frac{2}{f^{2}}\left[\frac{r-t_{0}}{2}\left(M_{1}-t_{0}\right)^{2}-\frac{1}{6}\left(M_{1}-t_{0}\right)^{3}+\frac{1}{6}\left(M_{1}-r\right)^{3}\right]$ \\
\hline & & & $t_{0} \leq M_{1}<r$ & $\frac{1}{f^{2}}\left[\left(r-t_{0}\right)\left(M_{1}-t_{0}\right)^{2}-\frac{1}{3}\left(M_{1}-t_{0}\right)^{3}\right]$ \\
\hline \multirow{2}{*}{$t_{0}, t_{0}+r$} & \multirow{2}{*}{$t_{0}+r, 2 t_{0}+r$} & \multirow{2}{*}{$2 t_{0}+r-t_{2}$} & $M_{1} \geq t_{0}$ & 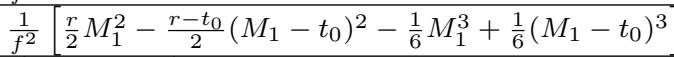 \\
\hline & & & $M_{1}<t_{0}$ & $\frac{1}{f^{2}}\left(\frac{r}{2} M_{1}^{2}-\frac{1}{6} M_{1}^{3}\right)$ \\
\hline$\left[t_{0}, t_{0}+r\right)$ & {$\left[2 t_{0}+r, f\right)$} & 0 & $\sim$ & $\frac{M_{1} r s}{f^{2}}$ \\
\hline
\end{tabular}

TABLE I: Case analysis for expected transmission times

client to hotspot mode with an overhead of $t_{0}$ seconds, stays in hotspot mode for $r$ seconds, switches back to client mode with another $t_{0}$-second overhead, and then stays in client mode for $s$ seconds, so on and so forth. Phones can retrieve the optimal mode-switching parameters from a central server.

For simplicity we assume that the hotspot-to-client and client-to-hotspot switching overheads are the same, confirmed by our experiments. Given the previous description, the switching procedure repeats with a period of $2 t_{0}+r+s$ seconds. Assuming the vehicle-vehicle and vehicle-AP meeting rates are $\beta$ and $\gamma$, respectively $(\beta+\gamma=1)$, we have the following optimization objective function,

$$
F(\beta, \gamma)=\max _{r, s}\left(\beta \cdot T_{1}+\gamma \cdot T_{2}\right),
$$

in which $T_{1}$ and $T_{2}$ are the expected phone-to-phone and phone-to-AP transmission durations, respectively. The optimization is over design parameters $r$ and $s$. Other parameters are not design parameters.

Let the base function $f(t)$ be a periodic function with period $f=2 t_{0}+r+s$,

$$
f(t)= \begin{cases}0, & 0 \leq t \leq t_{0} \\ 1, & t_{0}<t \leq t_{0}+r \\ 0, & t_{0}+r<t \leq 2 t_{0}+r \\ -1, & 2 t_{0}+r<t \leq 2 t_{0}+r+s\end{cases}
$$

When $f(t)=0$, the phone is switching between modes; $f(t)=1$ indicates that the phone is in hotspot mode, and $f(t)=-1$ client mode. In our calculation, we assume that the switching overhead does not dominate either of the actual mode durations, i.e., $r, s>t_{0}$.

Upon entering the communication range of each other, the two vehicles $v_{1}$ and $v_{2}$ are at $t_{1}$ and $t_{2}$ withint their respective base function $f(t)$ periods, $0 \leq t_{1}, t_{2}<2 t_{0}+r+s$. We describe the switching patterns of $v_{1}$ and $v_{2}$ as $f_{1}(t)=f\left(t+t_{1}\right)$ and $f_{2}(t)=f\left(t+t_{2}\right), t \in\left[0,2 t_{0}+r+s\right)$, respectively. We then define $t^{*}$ to be the time since meeting that the phones in two cars establish connection. It follows that,

$$
t^{*}=\min _{t}\left\{t: f_{1}(t) f_{2}(t)<0\right\}
$$

Since two cars can meet at any time, we consider $t_{1}$ and $t_{2}$ to be uniformly distributed over $\left[0,2 t_{0}+r+s\right)$. We use $M_{1}$ to denote the total time duration in which the two cars $v_{1}$ and $v_{2}$ are within communication range with each other. It then follows that the two phones can only establish connection if $M_{1} \geq t^{*}$. With these notations, we derive the analytic formula of the expected transmission time

$$
T_{1}=E_{t_{1}, t_{2}}\left[M_{1}-t^{*}\right]
$$

which can be computed generically as

$$
\frac{1}{f^{2}} \int_{t_{1}} \int_{t_{2}}\left(M_{1}-t^{*}\right) d t_{2} d t_{1}
$$

Now we compute the actual analytical expression for $T_{1}$ for the various cases of $t_{1}$ and $t_{2}$ value ranges.

(I) We first consider the case where $t_{1}, t_{2} \in\left[0, t_{0}\right)$. It's easily seen that $f_{1}(t) f_{2}(t) \geq 0$ for any $t$. Therefore, $E\left(M_{1}-t^{*}\right) I_{\left(t_{1} \in\left[0, t_{0}\right)\right)} I_{\left(t_{2} \in\left[0, t_{0}\right)\right)}=0$. Note that the $I$ 's are just indicator functions, which we omit in writing for the rest of the derivations.

(II) Next we consider the case where $t_{1} \in\left[0, t_{0}\right), t_{2} \in$ $\left[t_{0}, t_{0}+r\right)$. Analyzing the physical process, we have $t^{*}=$ $2 t_{0}+r-t_{2}$ if and only if $t_{2}-t_{1} \geq t_{0}$. Then, if $M_{1} \geq t_{0}+r \geq t^{*}$, we have

$$
\begin{aligned}
& E\left(M_{1}-t^{*}\right)=\frac{1}{f^{2}} \int_{t_{1}} \int_{t_{2}}\left(M_{1}-t^{*}\right) d t_{2} d t_{1} \\
= & \frac{1}{f^{2}} \int_{0}^{t_{0}} \int_{t_{1}+t_{0}}^{t_{0}+r}\left(M_{1}-2 t_{0}-r+t_{2}\right) d t_{2} d t_{1} \\
= & \frac{1}{2 f^{2}}\left[\left(M_{1}-t_{0}\right)^{2} t_{0}-\frac{1}{3}\left(M_{1}-r\right)^{3}+\frac{1}{3}\left(M_{1}-t_{0}-r\right)^{3}\right] .
\end{aligned}
$$

Alternatively if $r \leq M_{1}<t_{0}+r$, to ensure $M_{1} \geq t^{*}=$ $2 t_{0}+r-t_{2}$, we need $t_{2} \geq 2 t_{0}+r-M_{1}$. Now $t_{2}$ has two possible lower bounds, $t_{1}+t_{0}$ and $2 t_{0}+r-M_{1}$. If $t_{1}+t_{0}>2 t_{0}+r-M_{1}$, it can be inferred that $t_{1}>t_{0}+r-M_{1}$, then,

$$
\begin{aligned}
& E\left(M_{1}-t^{*}\right)=\frac{1}{f^{2}} \int_{t_{1}} \int_{t_{2}}\left(M_{1}-t^{*}\right) d t_{2} d t_{1} \\
= & \frac{1}{f^{2}} \int_{t_{0}+r-M_{1}}^{t_{0}} \int_{t_{1}+t_{0}}^{t_{0}+r}\left(M_{1}-2 t_{0}-r+t_{2}\right) d t_{2} d t_{1} \\
= & \frac{1}{2 f^{2}}\left[\left(M_{1}-t_{0}\right)^{2}\left(M_{1}-r\right)-\frac{1}{3}\left(M_{1}-r\right)^{3}\right] .
\end{aligned}
$$

On the other hand if $t_{1}+t_{0}<2 t_{0}+r-M_{1}$, it can be inferred that $t_{1}<t_{0}+r-M_{1}$, then,

$$
\begin{aligned}
& E\left(M_{1}-t^{*}\right)=\frac{1}{f^{2}} \int_{t_{1}} \int_{t_{2}}\left(M_{1}-t^{*}\right) d t_{2} d t_{1} \\
= & \frac{1}{f^{2}} \int_{0}^{t_{0}+r-M_{1}} \int_{2 t_{0}+r-M_{1}}^{t_{0}+r}\left(M_{1}-2 t_{0}-r+t_{2}\right) d t_{2} d t_{1} \\
= & \frac{1}{2 f^{2}}\left(M_{1}-t_{0}\right)^{2}\left(t_{0}+r-M_{1}\right) .
\end{aligned}
$$

Adding these two together, we thus have,

$$
E\left(M_{1}-t^{*}\right)=\frac{1}{2 f^{2}}\left[\left(M_{1}-t_{0}\right)^{2} t_{0}-\frac{1}{3}\left(M_{1}-r\right)^{3}\right] .
$$




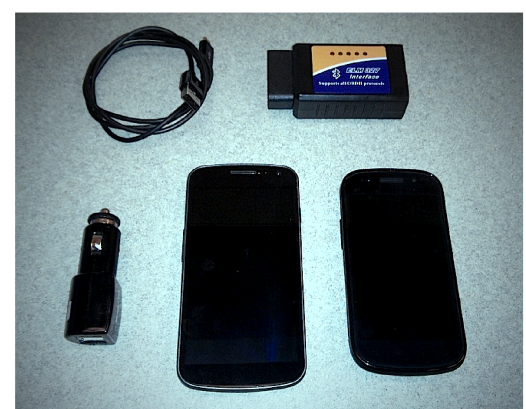

(a) Hardware components of our prototype system

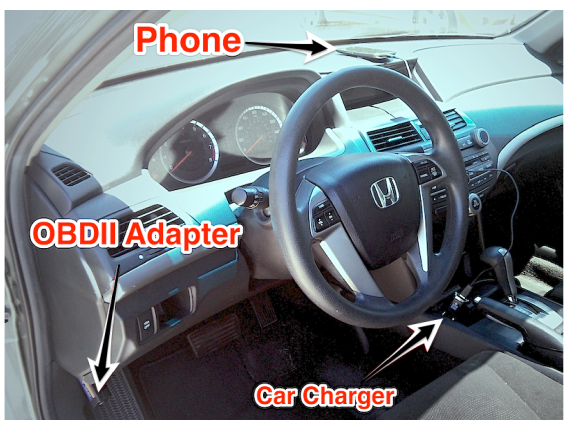

(b) Our system as installed in a vehicle

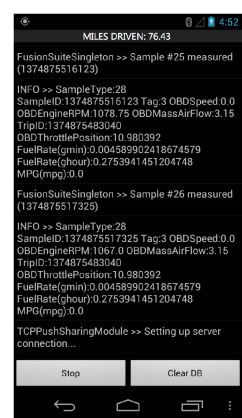

(c) System running screenshot

Fig. 4: Hardware components, installation, and running screenshot of our prototype system

If $t_{0} \leq M_{1}<r$, to ensure $M_{1} \geq t^{*}=2 t_{0}+r-t_{2}$, we have $t_{2} \geq 2 t_{0}+r-M_{1} \geq t_{1}+t_{0}$, then

$$
\begin{aligned}
& E\left(M_{1}-t^{*}\right)=\frac{1}{f^{2}} \int_{t_{1}} \int_{t_{2}}\left(M_{1}-t^{*}\right) d t_{2} d t_{1} \\
= & \frac{1}{f^{2}} \int_{0}^{t_{0}} \int_{2 t_{0}+r-M_{1}}^{t_{0}+r}\left(M_{1}-2 t_{0}-r+t_{2}\right) d t_{2} d t_{1} \\
= & \frac{t_{0}}{2 f^{2}}\left(M_{1}-t_{0}\right)^{2} .
\end{aligned}
$$

We omit derivation details for the rest of the cases and collect results for all cases in Table I.

Since $\left[0, r+t_{0}\right)$ and $\left[r+t_{0}, f\right)$ are symmetric, we have

$$
\begin{aligned}
T_{1}= & E\left[M_{1}-t^{*}\right] \\
= & \frac{2 r s}{f^{2}} M_{1}+I_{\left(M_{1}<t_{0}\right)} f_{1}\left(r, s, M_{1}\right)+I_{\left(M_{1} \geq t_{0}\right)} f_{2}\left(r, s, M_{1}\right) \\
& +I_{\left(t_{0} \leq M_{1}<r+t_{0}\right)} f_{3}\left(r, s, M_{1}\right)+I_{\left(M_{1} \geq r+t_{0}\right)} f_{4}\left(r, s, M_{1}\right) \\
& \quad+I_{\left(t_{0} \leq M_{1}<s+t_{0}\right)} f_{5}\left(r, s, M_{1}\right)+I_{\left(M_{1} \geq s+t_{0}\right)} f_{6}\left(r, s, M_{1}\right),
\end{aligned}
$$

where

$$
\begin{aligned}
f_{1}\left(r, s, M_{1}\right)= & \frac{s+r}{f^{2}} M_{1}^{2} \\
f_{2}\left(r, s, M_{1}\right)= & \frac{1}{f^{2}}\left[\frac{2}{3} M_{1}^{3}-\frac{2}{3}\left(M_{1}-t_{0}\right)^{3}-2 t_{0}\left(M_{1}-t_{0}\right)^{2}\right. \\
& \left.+2 M_{1} t_{0}(r+s)+\frac{4}{3} t_{0}^{3}-\left(2 M_{1}+r+s\right) t_{0}^{2}\right] \\
f_{3}\left(r, s, M_{1}\right)= & \frac{1}{f^{2}}\left[r\left(M_{1}-t_{0}\right)^{2}-\frac{1}{3}\left(M_{1}-t_{0}\right)^{3}\right] \\
f_{4}\left(r, s, M_{1}\right)= & \frac{1}{f^{2}}\left[r\left(M_{1}-t_{0}\right)^{2}+\frac{1}{3}\left(M_{1}-t_{0}-r\right)^{3}\right. \\
& \left.-\frac{1}{3}\left(M_{1}-t_{0}\right)^{3}\right] \\
f_{5}\left(r, s, M_{1}\right)= & \frac{1}{f^{2}}\left[s\left(M_{1}-t_{0}\right)^{2}-\frac{1}{3}\left(M_{1}-t_{0}\right)^{3}\right] \\
f_{6}\left(r, s, M_{1}\right)= & \frac{1}{f^{2}}\left[s\left(M_{1}-t_{0}\right)^{2}+\frac{1}{3}\left(M_{1}-t_{0}-s\right)^{3}\right. \\
& \left.-\frac{1}{3}\left(M_{1}-t_{0}\right)^{3}\right] .
\end{aligned}
$$

Note that the conditions in the above equations are not mutually exclusive, which, however, does not affect the optimization.

For modeling phone-to-AP communication, assume that there is a WiFi AP always in hotspot mode. A vehicle comes in range of the AP and stays in range for $M_{2}$ seconds. With the same notations as above, we have

$$
t^{*}=\left\{\begin{array}{rl}
t_{0}-t_{1}, & t_{1} \in\left[0, t_{0}\right) \\
0, & t_{1} \in\left[t_{0}, t_{0}+r\right) \\
t_{0}+s+\left(2 t_{0}+r-t_{1}\right), & t_{1} \in\left[t_{0}+r, 2 t_{0}+r\right) \\
t_{0}+\left(f-t_{1}\right), & t_{1} \in\left[2 t_{0}+r, f\right)
\end{array} .\right.
$$

Therefore, the expected connection time to a WiFi AP is,

$$
\begin{aligned}
T_{2}= & E\left(M_{2}-t^{*}\right)=\frac{M_{2} r}{f}+I_{\left(M_{2}<t_{0}\right)} \frac{M_{2}^{2}}{2 f} \\
& +I_{\left(M_{2} \geq 0\right)} \frac{1}{2 f}\left[M_{2}^{2}-\left(M_{2}-t_{0}\right)^{2}\right] \\
& +I_{\left(t_{0} \leq M_{2}<f-r\right)} \frac{1}{2 f}\left(M_{2}-t_{0}\right)^{2} \\
& +I_{(M-2 \geq f-r)} \frac{1}{2 f}\left[\left(M_{2}-t_{0}\right)^{2}-\left(M_{2}-f+r\right)^{2}\right] .
\end{aligned}
$$

Again, the conditions in the above equation is not mutually exclusive.

Given the values of $t_{0}, M_{1}, M_{2}, \beta$ and $\gamma$, we can then solve $F(\beta, \gamma)$ using off-the-shelf non-linear optimization solvers. The evaluation of our solution is presented in Section VI.

\section{System Design}

In this section, we give an overview of our vehicular phoneto-phone communication system, and discuss in detail a few important design issues.

\section{A. System Overview}

Shown in Figure 4(b) is our prototype system as installed in a vehicle. A close-up shot of the various hardware components used in our system is shown in Figure 4(a). The Android phone (Galaxy Nexus [32] or Nexus S [33]) is placed under the windshield of the vehicle and is connected to the car charger. ${ }^{1}$ The prototype application running on the phones collects and shares various driving data, including GPS trajectories, car engine OBD (onboard diagnostic) readings [34], as well as motion (accelerometer and gyroscope) data traces. The collecting and sharing of such location, car engine and motion data exemplifies a participatory sensing app that has a focus on how people's driving patterns and habits affect their vehicles' fuel consumptions. Our prototype system operates in completely autonomous manners, needing no human intervention.

The GPS, accelerometer, and gyroscope data traces are collected from the phone's corresponding built-in sensors. The engine OBD data is read using the ELM 327 OBD-tobluetooth adapter plugged into the car's OBD-II port, and then transmitted to the phone via bluetooth.

All collected sensory data is temporarily stored locally on the phone in a database. Whenever an available WiFi AP is detected in range, data is offloaded to the back-end server and then deleted from the phone's local storage. When two

\footnotetext{
${ }^{1}$ Please note that in this paper we do not consider phone's battery consumption as it can be plugged into the car charger during in-vehicle operation.
} 
vehicles are i) within the communication range of each other, and ii) in compatible modes (i.e., one as a hotspot and the other client), they exchange data until they move out of each other's communication range or complete sharing all their stored data.

\section{B. Design Issues}

We next discuss several important issues in our system design: 1) Adaptive system update; 2) which transport layer protocol to use; 3) scheduling during data transfer; 4) Multivehicle communication policy; and 5) learning WiFi AP maps.

1) Adaptive System Updates: In our targeted vehicular scenarios, phones enter and leave communication ranges with each other or WiFi APs from time to time. Therefore, the parameters $M_{1}$ and $M_{2}$ are not unknown or fixed, especially when the system starts running with no available historical data. We explain here how these parameters are computed and updated dynamically as the system evolves.

We treat $\beta, \gamma, M_{1}$ and $M_{2}$ as random variables. Then the optimal values of $r$ and $s$ are

$$
\left\langle r^{*}, s^{*}\right\rangle=\arg \max _{r, s} E_{M_{1}, M_{2}}\left[E(\beta) T_{1}+E(\gamma) T_{2}\right] .
$$

Initially the distributions of these parameters are unknown. We therefore just make an initial guess at this stage. Then as the system runs, detailed data of the parameters are sent back and the corresponding empirical distribution are updated. The parameters $M_{1}$ and $M_{2}$ represent the time durations of two cars, or a car and a WiFi AP, being within communication range of each other, respectively. Thus, they are a function of car velocity and distance, which is a known constant. The expectations of $\beta$ and $\gamma$ can be estimated by $N_{c} /\left(N_{c}+N_{w}\right)$ and $N_{w} /\left(N_{c}+N_{w}\right)$, where $N_{c}$ is the total number of vehicleto-vehicle meeting events and $N_{w}$ vehicle-to-AP. Whenever a phone-to-phone connection or a phone-to-AP connection is established, the velocities of the vehicles are transmitted and eventually will reach the back-end server. As this information accumulates, the empirical distributions of the parameters is updated. Consequently, a new $\left\langle r^{*}, s^{*}\right\rangle$ is generated and then sent back to the vehicles. As data accumulates, by the law of large number, the empirical distributions converge to the true distributions of these parameters, therefore, $\left\langle s^{*}, t^{*}\right\rangle$ will asymptotically lead to optimal system performance.

To disseminate the updated parameters into the network, we allow phones to receive this information via the cellular data channel. As the amount of data needed for this is negligible compared to other mobile sensing data, the whole network is updated immediately with only a tiny extra cost.

2) Transport Layer Protocol: TCP and UDP have their own strengths and weaknesses. To decide which one to use, we conduct a series of experiments to compare their performance in our system. During each experiment, two vehicles start at two ends of a long street, and move toward each other at fixed speeds until they reach the other end of the street. One phone serves as the hotspot and the other client. The client continuously sends data packets to the hotspot after connecting to it upon entering communication range. Packet sequence numbers are used to simulate sensory data for transmissions. TCP and UDP communications are measured separately. In addition, we optimize the TCP real-time responses to improve system efficiency by turning off the Nagle's Delay option [35], which is used to purposefully delay transmission, increasing bandwidth at the expense of latency. The packet reception ratio
(PRR) under varying car speeds, ranging from 10 to $30 \mathrm{mph}$, is recorded. The experiments are repeated on different streets to minimize the effect of external noise.

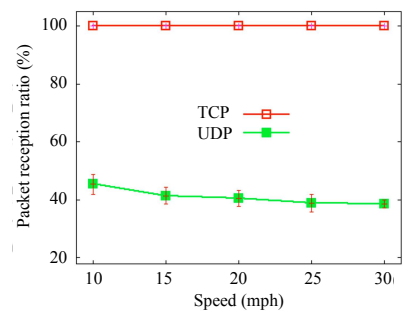

(a) Packet reception ratio under varying speeds (TCP/UDP)

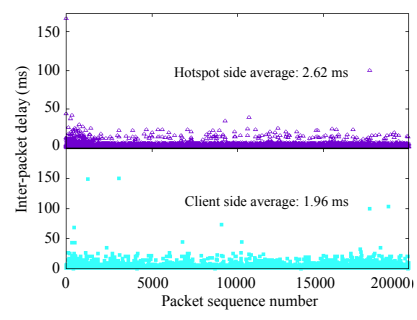

(b) Pattern of inter-packet latency on both hotspot and client sides.
Fig. 5: Empirical results for design decisions.

Results are shown in Figure 5(a), from which we see that UDP results in significant packet losses, only receiving about $40 \%$ of packets on average under all speeds. We also measure the PRR in the stationary case when two vehicles are parked near each other. We find that packet losses rarely occur, implying that the losses are mainly due to unreliable wireless links in mobile situations. Also, we measure that the inter-packet latency is around 1.5 millisecond for UDP and 1.9 millisecond for TCP under all speeds. This result indicates that the data transfer efficiency of TCP is comparable to UDP when the Nagle's Delay option is turned off, and the number of packets received using UDP is only $51 \%$ of the number using TCP, thus TCP results in a more efficient data transmissions. Therefore, we chose to use TCP in our system.

3) Scheduling During Data Transfer: Upon establishing connection between two phones, we can either schedule the transfers to take place in a serial manner (e.g., A sends to B, then B sends to A), or have them in parallel, i.e., two separate threads on each phone, one for sending and the other receiving, so the OS takes care of the lower-level scheduling. The former approach achieves controlled scheduling, but it is difficult to decide an appropriate time slot value, and introduces switching overhead. For the latter, we need to check whether transmission performance is affected and fairness provided. Therefore, we repeat the two-vehicle experiment with the latter approach. The inter-packet delay times on both sides is shown in Figure 5(b). We see that the data transfer with two threads is reasonably fair for both directions - the average inter-packet latency at the hotspot side is $2.62 \mathrm{~ms}$, only slightly higher than the $1.96 \mathrm{~ms}$ measured at the other side. Considering that the inter-packet delay is 1.9 millisecond when transferring in one direction, using two separate threads can improve the transmission efficiency by $69.5 \%$. Thus we choose this approach in our system design.

4) Multi-Vehicle Communication Policy: We briefly talk about the scenario under which multiple vehicles are within communication range of each other. In our smartphone-based vehicular sensing system, let's assume they form a startopology network, the hotspot acts as the center and other clients connect to it. The number of clients is limited by the capacity of the hotspot (e.g., the maximum number of connections for iPhone 5 is five, as confirmed by AT\&T and Verizon). The hotspot communicates to its clients simultaneously via multiple threads, and data from one client flows to others through the hotspot. 


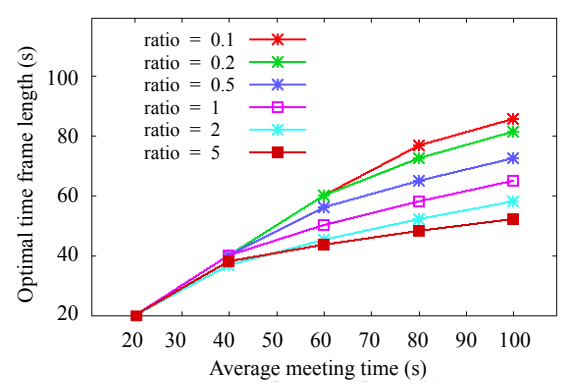

(a) Optimal time frame length

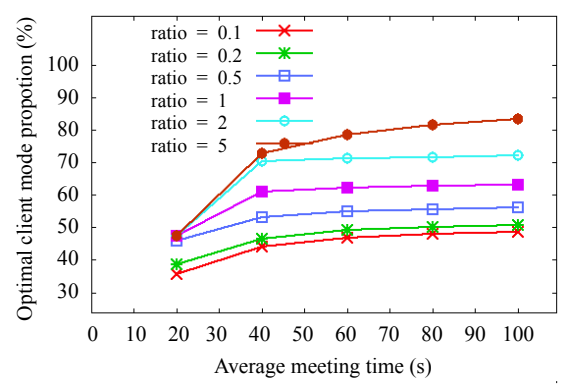

(b) Optimal client mode proportion

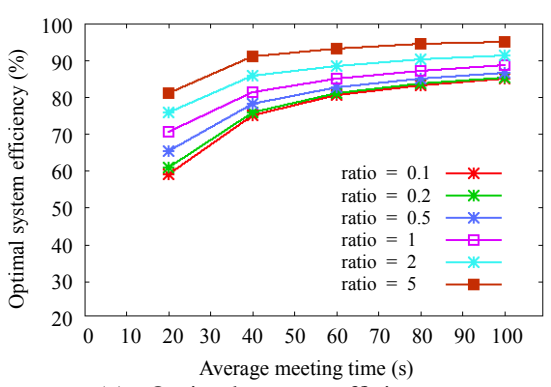

(c) Optimal system efficiency

Fig. 6: Results under varying vehicle-AP/vehicle-vehicle meeting time durations and ratios

One choice in the multi-vehicle scenario is to switch their roles dynamically for better global communication opportunity. For instance, a client $A$ connects to a hotspot $B$, and they start transmitting data. Then $A$ notices that another three hotspots, $C, D$, and $E$, appear in its wifi list. Hence, the best way for this local area network is to switch $A$ as a hotspot, and the other four phones as clients, so as to get everyone involved in communication. However, this approach suffers from two main drawbacks. First, it requires an extra switch time for the client (e.g., $A$ ) to notify each hotspot to switch to client and then switch itself into a hotspot. Second, the mobility of these vehicles are undeterministic, thus it is hard to judge whether this switching process is worthy in general. Therefore, we decide not to support multi-vehicle communication in our system, as also reasoned about in Section III.

5) WiFi AP Maps: We also recognize that having prior knowledge about WiFi AP maps could help optimize our system. Several such maps exist, being managed by the government [36] and wireless operators [37]. However, the availability of these maps is a big challenge since they are typically not made pubic. What's more, WiFi APs are generally designed to cover indoor environments (e.g., Cafe, office, etc) and thus are not largely accessible in vehicular settings. One possibility is to let participants' phones record local WiFi AP information and share to the central server. The server can then derive a global map and broadcast it back to all participants. This approach, however, is problematic as different participants may have different accesses to different APs. This may lead to inaccurate estimations. In addition, the highly mobile vehicular environment can lead to unstable communication patterns and subsequent conflicting results on the central server. We thus decide not to assume the availability of WiFi AP map information in this paper.

\section{Evaluation}

Having presented our analytical model and discussed system design details, we, in this section, evaluate the performance of our automatic phone-to-phone communication scheme for vehicular networking applications. We report findings from our campus-wide deployment, and present optimization results through simulation experiments using a larger-scale real-world taxicab dataset.

\section{A. Experiment Setup}

We conducted a human subject study. ${ }^{2} 35$ people participated (university faculty, staff, and students of both genders, ranging from early 20 s into late 40 s, from various departments)

\footnotetext{
${ }^{2}$ The study was conducted under IRB protocol \#10092.
}

averaging 2 weeks each, and collectively drove for around 4,000 miles. While we expect a mobile sensing application to run on participants' own phones, in our study we gave people phones pre-loaded with our test application. A mixture of both Galaxy Nexus and Nexus S phones were given to participants to be installed in their own vehicles. No specific driving routes were pre-selected; all participants were asked to drive normally and carry out their daily routines as usual. Comprehensive logging information was displayed on the phone during the running of the system, as illustrated in Figure 4(c), to notify the participants of the status of the system if they were interested.

TCP communication is used with Nagle's Delay disabled, as we learn from our prior tests that having this option enabled has negative impact on communication throughput. The switching overhead is estimated to be at about 3.5 seconds for Galaxy Nexus and 6.9 seconds for Nexus S phones. During the data transfer process, two separate threads are spawn concurrently, one for sending and the other receiving. Fifty consecutive data samples are combined into one larger packet before sending in order to improve throughput.

\section{B. Experiment Results}

We estimate the values of $t_{0}, M_{1}, M_{2}, \beta$ and $\gamma$ from data collected in our deployment, and investigate the relationship between optimal parameters and various system coefficients, under varying average meeting time durations $\left(M_{1}, M_{2}\right)$ and vehicle-AP to vehicle-vehicle meeting ratios $\left(\frac{\gamma}{\beta}\right)$.

Figure 6(a) shows how optimal time frame lengths $\left(2 t_{0}+\right.$ $r+s)$ are affected by meeting times and ratios. We see that, when the average meeting time is below 40s, meeting ratios have little effect on optimal time frame lengths. When the average meeting time increases beyond 40s, the optimal frame lengths differ considerably as the meeting ratio variesthe more dominant vehicle-AP meetings are (as opposed to vehicle-vehicle meetings), the shorter the optimal frame lengths become. We also notice that as the average meeting time increases beyond 40s, the growth of the optimal frame length slows down.

Figure 6(b) illustrates how optimal client mode proportion $\left(\frac{s}{2 t_{0}+r+s}\right)$ changes with different meeting times and ratios. We observe that, as the vehicle-vehicle meeting ratio decreases, the optimal client proportion increases. In particular, when vehicle-vehicle meetings are about 10 times that of vehicle-AP ones, the hotspot and client proportions are roughly the same with each other; On the other hand, the optimal client mode proportion increases beyond $80 \%$ when vehicle-AP meetings become dominant. These results suggest the following, i) In a dense vehicular network, in order to achieve the highest 


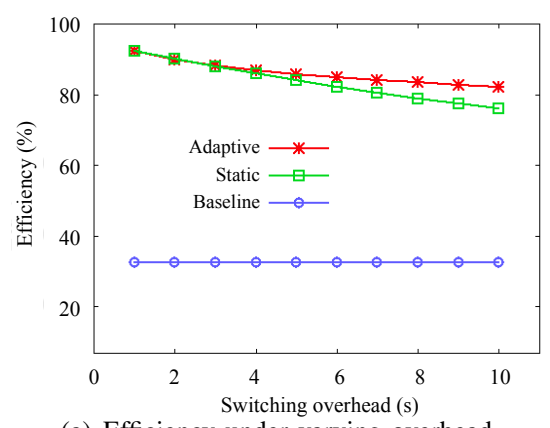

(a) Efficiency under varying overhead

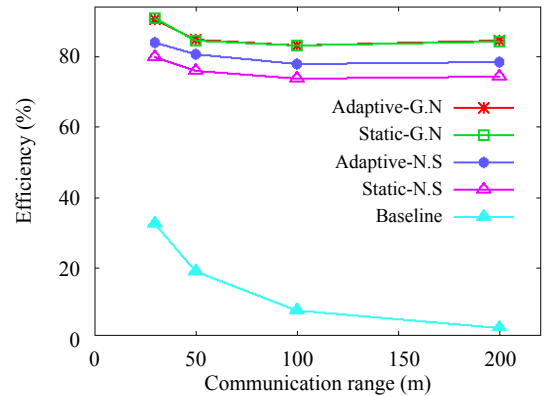

(b) Efficiency under varying range

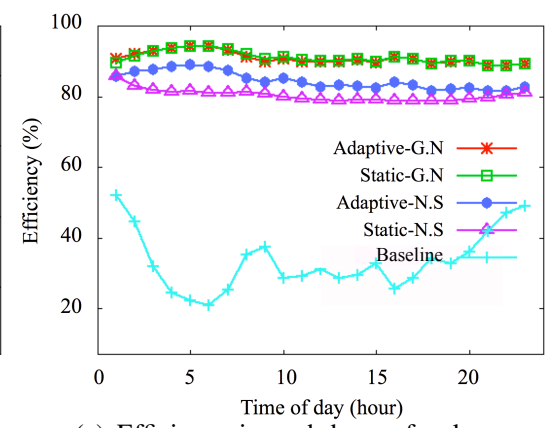

(c) Efficiency in each hour of a day

Fig. 7: Experimental results of system efficiency.

system efficiency, phones should spend approximately the same amount of time in hotspot and client modes; and ii) In a sparse vehicular network, phones should stay in client mode as much as possible in order to maximize the probability of communicating with WiFi APs. We can also easily see from the figure that the optimal client mode proportion increases when the average meeting time lengthens.

Figure 6(c) shows the optimal estimated system efficiency $\left(\beta \frac{T_{1}\left(r^{*}, s^{*}\right)}{M_{1}}+\gamma \frac{T_{2}\left(r^{*}, s^{*}\right)}{M_{2}}\right.$, note that $T_{1}$ and $T_{2}$ are functions that take the optimal parameters $r^{*}, s^{*}$ as inputs) varies with meeting times and ratios. We see that the efficiency increases monotonically with both the average meeting time and vehicleAP meeting ratio.It is quite promising that data communication takes up over $55 \%$ of meeting times in almost all cases, and even reaching above $90 \%$ in certain cases (higher vehicle-AP meeting ratio and long meeting time).

\section{Larger-Scale Simulation Results}

Our deployment and human subject study help us get initial ideas of how our proposed system behaves. To analyze the system performance in a much larger scale, we turn to simulation experiments using the T-drive real-world taxicab dataset [29], [30], which contains the GPS trajectories of 10,357 taxicabs during the period of Feb. 2nd through Feb. 8th, 2008 in Beijing. To better represent our mobile sensing application scenario, we select the central part of city and filter out the suburb area where vehicles are sparse. Thus our experiments contain 9,211 taxicabs, covering the central Beijing area. We focus on evaluating the system efficiency of our proposed optimization approach in this set of larger-scale simulation experiments.

We assume that $10 \%$ of this area is covered by WiFi APs to measure the performance of offloading events. This number is motivated by results from other large cities such as San Francisco and Seattle [38]. These WiFi APs are spread out equally in the central part of the area.

The communication range of WiFi APs and taxicabs are set to 250 and 30 meters, respectively. Both are based on our actual measurements. We also experiment with the situations where the taxicabs' communication range varies from $30 \mathrm{~m}$ to $50 \mathrm{~m}, 100 \mathrm{~m}$, and $200 \mathrm{~m}$, in order to investigate the cases where the next generation phones are more powerful and capable of achieving larger communication ranges. Other system settings and parameters, including data generation and offload process, follow that of our small-scale deployment study.

We carry out the simulation using the T-drive dataset as follows. For the first 24-hour's data, we first extract meeting intervals by recording all vehicle-vehicle and vehicle-AP pairs that are in communication range at each time point, then compute the optimal parameters based on the analytical model discussed in Section IV. Finally we apply these parameters to the meeting intervals and calculate the overall system efficiency under three different candidate approaches: Adaptive, Static, and Baseline. Adaptive updates system parameters every hour based on historical data and applies them to all vehicles in the network. Static only uses the data from the first hour to calculate the optimal parameters, and then remains the same during the whole process. Baseline considers the baseline case in which phone-to-phone communication is not enabled.

We first investigate how system efficiency changes as the switching overhead $\left(t_{0}\right)$ varies. Figure 7(a) shows the results with the mode switching overhead ranging from $1 \mathrm{~s}$ to $10 \mathrm{~s}$, where phones' communication range is set to be $30 \mathrm{~m}$. We see that the system efficiency for Adaptive performs slightly better than that of Static and is over $80 \%$ for all cases, specifically, $90 \%$ and $90 \%$ for Nexus $4\left(t_{0}=2.1\right.$ seconds), $88 \%$ and $86 \%$ for Galaxy Nexus ( $t_{0}=3.5$ seconds), and $84 \%$ and $79 \%$ for Nexus $\mathbf{S}\left(t_{0}=6.9\right.$ seconds). This indicates that our proposed solution can achieve high system efficiency using off-theshelf smartphones and thus is highly practical. Also, since the Baseline approach does not allow phone-phone communication functionality at all, the system efficiency remains at $33 \%$, which is just the ratio of overall phone-AP to all meeting time.

Figure 7(b) shows the system efficiency under varying phone communication ranges when the mode switching overhead is 3.5 and 6.9 seconds, to emulate the use of Galaxy Nexus (G.N) and Nexus S (N.S) phones. We see that the efficiency of both Adaptive and Static does not change much as the transmission range increases. The efficiency of Baseline decreases as transmission range goes up. We also notice that, again, the system efficiency for Adaptive performs only slightly better than Static for both phones. This suggests that in a relatively dense vehicular network setting, our proposed solution quickly converges to optimal system parameters and does not need extensive training phase.

We next study the time-of-day system efficiency in an hourby-hour fashion. As Figure 7(c) shows, the system efficiency measurements for both Adaptive and Static do not change much throughout the day, implying that both approaches work quite well consistently. On the other hand, we see that the Baseline approach leads to large oscillations, mainly due to the shift of traffic patterns throughout the day, with a higher vehicle-AP meeting ratio in the evening. Therefore, our proposed approach, be it Adaptive or Static, delivers a much more stable and 
predictable system performance than the baseline.
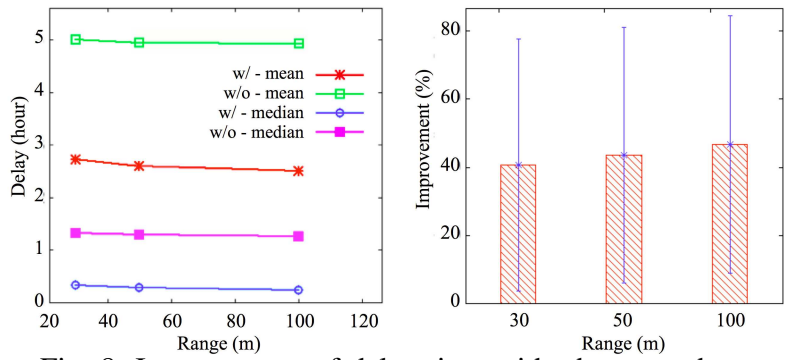

Fig. 8: Improvement of delay time with phone-to-phone communication.

Finally, Figure 8 shows the application-level benefit that direct phone-to-phone communication brings about. We record the delay time of delivery for data generated in the first hour by all 9,211 taxicabs, with and without our solution and under varying transmission ranges. The throughput of phone-to-phone communication bandwidth is set to $746.5 \mathrm{kbps}$, obtained from measurements in our deployment. As we can see, enabling phone-to-phone communication largely decreases the delay time of data delivery, by more than $40 \%$ and up to about $50 \%$ on average for all communication ranges tested. More concretely, our solution helps reduce the average delay time from 5.0 to 2.7 hours, and the median from 1.3 to 0.3 hour. In addition, as the transmission range increases, the improvement by our solution also increases because it leads to more occurrences of data transfers among taxicabs. These results indicate that direct phone-to-phone communication significantly improves data collection and sharing in vehicular networking applications.

\section{CONCLusions}

In this paper, we present the design, implementation, and evaluation of a novel optimized vehicular mobile system that leverages both phone-to-phone and phone-to-AP communications from vehicle-resident smartphones. Our proposed solution optimizes vehicle meeting communication efficiency, does not require any change to existing infrastructure, and is completely transparent to end users. Results from our 35vehicle 2-month campus-wide deployment and a large-scale real-world dataset simulation demonstrate that our approach significantly reduces data transfer delay time and maintains over $80 \%$ (90\% in certain cases) system efficiency. Given the popularity of smartphones and importance of vehicular networks, we believe that this work will motivate further research on leveraging human encounters in mobile sensing and networking applications.

\section{ACKNOWLEDGEMENT}

This work was supported by NSF grants CNS 1059294 , CNS 1040380, and CNS 1239483, and NSFC grant U1301256.

\section{REFERENCES}

[1] R. Yanggratoke, A. Azfar, M. J. P. Marval, and S. Ahmed, "Delay tolerant network on android phones: Implementation issues and performance measurements," JOC, vol. 6, no. 6, pp. 477-484, 2011.

[2] A. Sharma, V. Navda, R. Ramjee, V. N. Padmanabhan, and E. M. Belding, "Cool-tether: energy efficient on-the-fly wifi hot-spots using mobile phones," in CoNext, 2009.

[3] N. Thompson, S. C. Nelson, M. Bakht, T. Abdelzaher, and R. Kravets, "Retiring replicants: congestion control for intermittently-connected networks," in InfoCom, 2010.
[4] S. C. Nelson, M. Bakht, and R. Kravets, "Encounter-based routing in dtns," in InfoCom, 2009.

[5] N. Banerjee, M. D. Corner, and B. N. Levine, "Design and field experimentation of an energy-efficient architecture for dtn throwboxes," TON, vol. 18, no. 2, pp. 554-567, 2010.

[6] K. W. Chen, "Cafnet: A carry-and-forward delay-tolerant network," Ph.D. dissertation, MIT, 2007

[7] R. Crepaldi, R. Beavers, B. Ehrat, M. Jaeger, S. Biersteker, and R. Kravets, "Loadingzones: leveraging street parking to enable vehicular internet access," in CHANTS, 2012.

[8] R. Crepaldi, M. Bakht, and R. Kravets, "Quicksilver: application-driven inter-and intra-cluster communication in vanets," in MobiOpp, 2012.

[9] J. Zhao and G. Cao, "Vadd: Vehicle-assisted data delivery in vehicular ad hoc networks," Vehicular Technology, IEEE Transactions on, vol. 57, no. 3, pp. 1910-1922, 2008 .

[10] IDC. Worldwide smartphone market expected to grow 55\% in 2011 and approach shipments of one billion in 2015, according to IDC, "http: //www.idc.com/getdoc.jsp?containerId=prUS22871611," 2013.

[11] E. Miluzzo, N. D. Lane, K. Fodor, R. Peterson, H. Lu, M. Musolesi, S. B. Eisenman, X. Zheng, and A. T. Campbell, "Sensing meets mobile social networks: the design, implementation and evaluation of the cenceme application," in SenSys, 2008.

[12] J. Yang, S. Sidhom, G. Chandrasekharan, T. Vu, H. Liu, Y. C. abd M. Gruteser, and R. Martin, "Detecting driver phone use levering car speakers," in MobiCom, 2011.

[13] R. Ganti, N. Pham, H. Ahmadi, S. Nangia, and T. Abdelzaher, "Greengps: A participatory sensing fuel-efficient maps application," in Mobisys, 2010.

[14] S. Mathur, T. Jin, N. Kasturirangan, J. Chandrasekaran, W. Xue, M. Gruteser, and W. Trappe, "Parknet: drive-by sensing of road-side parking statistics," in MobiSys, 2010.

[15] R. K. Balan, K. X. Nguyen, and L. Jiang, "Real-time trip information service for a large taxi fleet," in MobiSys, 2011.

[16] P. Mohan, V. N. Padmanabhan, and R. Ramjee, "Nericell: rich monitoring of road and traffic conditions using mobile smartphones," in SenSys, 2008

[17] A. Thiagarajan, L. Ravindranath, K. LaCurts, S. Madden, H. Balakrishnan, S. Toledo, and J. Eriksson, "Vtrack: accurate, energy-aware road traffic delay estimation using mobile phones," in SenSys, 2009.

[18] B. Hull, V. Bychkovsky, Y. Zhang, K. Chen, M. Goraczko, A. Miu, E. Shih, H. Balakrishnan, and S. Madden, "Cartel: a distributed mobile sensor computing system," in SenSys, 2006.

[19] D. Yang, G. Xue, X. Fang, and J. Tang, "Crowdsourcing to smartphones: incentive mechanism design for mobile phone sensing," in MobiCom 2012

[20] H. Ahmadi, N. Pham, R. Ganti, T. Abdelzaher, S. Nath, and J. Han, "Privacy-aware regression modeling of participatory sensing data," in SenSys, 2010.

[21] H. Ahmadi, T. Abdelzaher, J. Han, R. Ganti, and N. Pham, "On reliable modeling of open cyber-physical systems and its application to green transportation," in ICCPS, 2011.

[22] Customers Angered as iPhones Overload AT\&T. NY Times, September 2009, "http://goo.gl/KtuInV," 2013.

[23] iPhone overload: Dutch T-Mobile issues refund after 3G issues. Ars Technica, July 2010, "http://goo.gl/6DCNYh," 2013.

[24] H. Liu, S. Hu, W. Zheng, Z. Xie, S. Wang, P. Hui, and T. Abdelzaher, "Efficient $3 \mathrm{~g}$ budget utilization in mobile participatory sensing applications," in InfoCom, 2013.

[25] The Code for the New wpa_supplicant, "https://github.com/kelvie/ android-wpa_supplicant," 2013.

[26] C. Foresman, "Wi-fi direct protocol to ease peer-to-peer wifi connections," 2009.

[27] Android issue 82: Support ad hoc networking, "http://code.google.com/ p/android/issues/detail?id=82," 2013 .

[28] S. Trifunovic, B. Distl, D. Schatzmann, and F. Legendre, "Wifi-opp: ad-hoc-less opportunistic networking," in CHANTS, 2011.

[29] J. Yuan, Y. Zheng, X. Xie, and G. Sun, "Driving with knowledge from the physical world," in $K D D, 2011$.

[30] J. Yuan, Y. Zheng, C. Zhang, W. Xie, X. Xie, G. Sun, and Y. Huang, "T-drive: driving directions based on taxi trajectories," in GIS, 2010.

[31] S. Nirjon, A. Nicoara, C.-H. Hsu, J. Singh, and J. Stankovic, "Multinets Policy oriented real-time switching of wireless interfaces on mobile devices," in RTAS, 2012.

[32] Galaxy Nexus, "http://goo.gl/zdd9f," 2013.

[33] Nexus S, "http://www.google.com/phone/detail/nexus-s," 2013.

[34] R. Birnbaum and J. Truglia, Getting to know OBD II. R. Birnbaum, 2001.

[35] L. L. Peterson and B. S. Davie, Computer networks: a systems approach Elsevier, 2007.

[36] Washington, DC Wi-Fi Hot Spot Map, "http://goo.gl/cqR8KA," 2013.

[37] AT\&T Wi-Fi Hot Spot locations, "http://goo.gl/DaOf99," 2013.

[38] A. Balasubramanian, R. Mahajan, and A. Venkataramani, "Augmenting mobile 3g using wifi," in MobiSys, 2010. 